Rev. Elev. Méd. vét. Pays trop., 1974, 27 (2) : 189-194

\title{
Les onchocercoses des bovins togolais à $O$. dukei et $O$. dermata
}

\author{
par J. BUSSIERAS $(*)$, E. AMEGEE $\left(^{*}\right)$ et O. BAIN $\left({ }^{* *}\right)$
}

\begin{abstract}
RESUME
Les bovins togolais sont très fréquemment atteints de deux onchocercoses à caractère nodulaire: une onchocercose musculaire et souscutanée, due à $O$. dukei Bain, Bussiéras et Amégee, 1974, et une onchocercose dermique, due à $O$. dermata Bain, Bussiéras et Amégee, 1974.
\end{abstract}

Les bovins africains (taurins et zébus) sont connus pour être très souvent atteints de deux onchocercoses :

- une onchocercose aortique, due à Onchocerca armillata RAILLIET et HENRY, 1909 ;

- et une onchocercose du ligament cervical et des articulations, due à $O$. gutturosa Neumann, 1910.

Ces deux parasites semblent infester la quasitotalité des bovins d'Afrique tropicale, bien que LE ROUX (9) remarque l'absence de $O$. armillata sur le bétail zambien autochtone.

Récemment a été observée en Afrique orientale une onchocercose dermique, localisée surtout au scrotum et à la mamelle, due à $O$. ochengi Bwangamoi, 1969 (4, 5).

Enfin, dans divers pays africains, ont été signalées des infestations à $O$. gibsoni Cleland et Johnston, 1910, mais il semble que dans la plupart des cas la preuve de l'identité des parasites n’ait pas été apportée.

(*) Ecole Inter-Etats des Sciences et Médecine Vétérinaires de Dakar.

(**) Zoologie (Vers), Muséum National d'Histoire Naturelle de Paris.
Or des études récentes $(2,1)$ ont montré qu'en Afrique occidentale, et plus particulièrement au Togo, les bovins présentent fréquemment deux autres onchocercoses, à localisations principales respectivement musculaire et dermique.

\section{L'ONCHOCERCOSE MUSCULAIRE A $O$. DUKEI}

\section{Historique}

En 1927-28, au Ghana (la o Gold Coast * de cette époque), W.P.B. BEAL (3) rapporte que le capitaine STEWART a observé une nouvelle onchocercose bovine. Celle-ci se traduit par la présence de nodules de la grosseur d'un pois, principalement localisés aux muscles intercostaux, aux muscles des membres antérieurs et postérieurs, et aux flancs; les lésions pouvaient facilement être confondues avec la ladrerie bovine (à Cysticercus bovis).

En 1928-29, le même W.P.B. BEAL précise que cette onchocercose se retrouve aussi bien chez les zébus que chez les taurins.

En 1929-30, J.L. STEWART (10) indique la fréquence de la localisation de cette onchocercose aux muscles pectoraux, et précise qu'à 
Accra, sur 559 bovins abattus, 72 étaient parasités, soit un taux d'infestation de 12,9 p. 100.

Dans l'intervalle, CAMERON (1928) (6), à qui des spécimens avaient été transmis, étudie les vers adultes (mais non les microfilaires) et estime qu'ils ne présentent que peu ou pas de différences avec $O$. volvulus de l'homme et O. gibsoni du bœuf; le même auteur, en 1951 (7) admet même que c'est $O$. volvulus de l'homme que l'on retrouve dans les muscles intercostaux des bovins.

Les mêmes parasites semblent avoir été retrouvés en Zambie, Rhodésie et Malawi par LE ROUX (1957) (9), qui remarque que tous les bovins infestés vivent dans des zones à simulies.

En 1971, VAN DEN HEEVER (8) signale en Afrique du Sud des lésions analogues dans le tissu conjonctif sous-cutané et les muscles des parties inférieures du thorax et de l'abdomen des bovins.

Enfin, en 1973, est entreprise l'étude helminthologique du parasite, tel qu'on le récolte au Togo; ceci permet d'établir qu'il s'agit d'une espèce nouvelle, Onchocerca dukei Bain, Bussiéras et Amégee, 1974.

Bien entendu, il n'est pas certain que toutes les observations précédentes se rapportent à la même espèce; nous remarquerons seulement que dans tous les cas les auteurs relèvent la grande ressemblance extérieure entre les nodules onchocerquiens et les lésions de la cysticercose bovine, ce qui montre que ces nodules sont tous de dimensions très voisines. En outre, les localisations dans la musculature sont généralement tout à fait comparables.

\section{Espèces affectées et répartition géographique}

Nous ne retiendrons que les cas où l'espèce $O$. dukei a véritablement pu être identifiée.

Toutes nos observations ont été faites dans des abattoirs du Togo (villes de Lomé, Sokodé, Lama-Kara, Mango, Dapango) et du Dahomey (Cotonou), les animaux positifs étant :

- des taurins, Bos taurus, de race Somba, originaires du Togo et du Dahomey;

- des zébus, Bos indicus, originaires de Haute-Volta et du Niger.

La zone de plus forte infestation est la région de Lama-Kara (Togo), où on compte parfois jusqu'à 90 p. 100 de carcasses parasitées, le plus souvent massivement.

\section{Les lésions}

\subsection{Aspect macroscopique}

Les nodules à $O$. dukei ont généralement une forme allongée, et mesurent en moyenne 5 - 15 $\times 5-6 \mathrm{~mm}$.

La section d'un nodule montre une paroi fibreuse, épaisse, avec une cavité centrale emplie de parasites et d'un magma puriforme jaunâtre.

\subsection{Localisations}

Les nodules sont trouvés surtout en abondance dans la moitié inférieure du tronc. Pour préciser les localisations, une vache abattue à Lama-Kara et infestée massivement, a été examinée et une demi-carcasse en a été totalement disséquée, ce qui a donné les résultats suivants :

\section{A. Nodules musculaires}

Tête (m. masticateurs, m. hyoïdiens)

Encolure (toutes portions)

Muscles dorso-lombaires

Thorax :

- intercostaux

- transverse du thorax

- grand dorsal et grand dentelé :

- partie supérieure

- partie inféricure

- pectoraux

Abdomen :

- flanc

- grand droit de l'abdomen
$+$
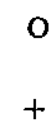

\section{o}

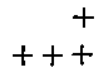

$+t$

$++$

$++$ 
Membre thoracique :

- m. scapulaires

- $\mathrm{m}$. brachiaux antérieurs

- m. brachiaux postérieurs (anconés)

- m. avant-bras

0
++
+
0

Membre pelvien :

- m. fessiers

- m. cruraux antérieurs

- $\mathbf{m}$. cruraux internes

- m. cruraux externes

- m. jambe

(ancones) 0

Cour

Diaphragme

$$
+
$$

$+$

$+$

o

o

0

$+$

B. Nodules sous-cutanés

Tête (joues)

Encolure

$++$

o

Tronc:

- moitié supérieure

$$
\begin{array}{r}
0 \\
+ \\
++ \\
++
\end{array}
$$

- moitié inférieure

- ombilic

- mamelle

- périnée

Membre thoracique

(surtout à la jonction avec l'encolure et avec le thorax):

- épaule

- bras

$+$

$+$

Membre pelvien :

- fesse

o

- cuisse

O

- jambe

o

\subsection{Etude microscopique}

L'examen du contenu des nodules montre la présence habituelle d'une femelle. Le plus souvent cette femelle est fécondée, contenant d'innombrables cufs et microfilaires, et elle est accompagnée de un, ou rarement deux ou trois, mâles. Certains nodules cependant ne contiennent aucun mâle mais seulement une femelle sans microfilaires.

Sur coupes histologiques, on constate que les nodules ne se développent pas dans les fibres musculaires, mais dans les éléments conjonctifs séparant les faisceaux musculaires. Leur coque est scléreuse, stratifiée, infiltrée de rares cellules inflammatoires, présentes surtout à la périphérie du nodule. Le magma central contient des cellules nécrosées.

\section{Mode de transmission}

Celui-ci est encore inconnu. I faut cependant observer :

- que la région de Lama-Kara, où prédo- mine cette onchocercose, est également la partie du Togo où l'onchocercose humaine est la plus fréquente;

- que LE ROUX (9) avait déjà remarqué qu'en Afrique orientale une onchocercose sans doute identique ne sévissait que dans les zones à simulies;

- que l'un de nous (E. AMEGEE) a capturé près de Lama-Kara des simulies non encore identifiées, gorgées sur bovins. Le tube digestif de l'une d'elle contenait trois microfilaires de $O$. dukei (ce qui ne prouve pas que le développement larvaire se serait poursuivi chez cette simulie).

Toutes ces raisons ne permettent évidemment pas d'affirmer le rôle vecteur des simulies.

\section{Discussion}

\subsection{Relations avec Onchocerca gibsoni}

Il existe une autre espèce d'onchocerque provoquant la formation de nodules dans le tissu 
conjonctif et parfois dans les muscles chez les bovins : Onchocerca gibsoni Cleland et Johnston, 1910, primitivement découverte en Australie.

$O$. gibsoni se distingue facilement de $O$. dukei par la morphologie des vers adultes $(2,1)$. En outre, les nodules à $O$. gibsoni sont souvent beaucoup plus volumineux, pouvant atteindre la grosseur d'un ouf de poule. Or la présence de $O$. gibsoni a été signalée plusieurs fois en Afrique tropicale, sans que soient toujours étudiés les parasites en cause; à l'avenir, il sera bon de préciser l'espèce parasitaire par une étude helminthologique.

\subsection{Confusions avec Cysticercus bovis}

Etant donné :

- la ressemblance superficielle des deux types de lésions (onchocercose musculaire et ladrerie);

- la grande fréquence de ces deux types d'infestations, au moins dans certaines contrées d'Afrique tropicale;

- le danger d'infestations humaines à partir des viandes ladres, alors que l'ingestion d'onchocerques est sans danger pour l'homme, il semble nécessaire, sur le plan pratique, de recommander un examen attentif des carcasses suspectes de ladrerie, avec incision des nodules pour vérifier le caractère vésiculaire de leur contenu. La distinction entre ladrerie sèche et onchocercose risquant d'être plus délicate, il peut être bon, dans ce cas, d'avoir recours à un examen microscopique; s'il s'agit d'onchocercose, on observe alors des fragments de femelle, et généralement d'innombrables microfilaires.

On remarquera aussi l'absence de localisation cardiaque de l'onchocercose.

\section{L'ONCHOCERCOSE DERMIQUE}

A O. DERMATA

La recherche des nodules à $O$. dukei localisés au tissu conjonctif sous-cutané, souvent adhérents à la face interne du cuir, a permis de mettre en évidence d'autres nodules, totalement intradermiques, qui contenaient une autre espèce d'onchocerques, Onchocerca dermata Bain, Bussiéras et Amégee, 1974.

\section{Répartition géographique et espèces affectées}

L'onchocercose dermique a été observée sur des taurins, Bos taurus, dans tous les abattoirs visités au Togo (Lomé, Sokodé, Lama-Kara, Mango, Dapango). Elle a été retrouvée aussi, à l'abattoir de Lomé, sur des zébus, Bos indicus, originaires de Haute-Volta.

\section{Les lésions}

\subsection{Aspect macroscopique}

Les nodules, intradermiques, ont des dimensions voisines de celles des lésions dues à $O$. dukei : 5 - $10 \times 4-7 \mathrm{~mm}$. Mais ils sont beaucoup moins visibles, dessinant simplement un relief sur la face interne du cuir, ou parfois même perceptibles seulement à la palpation.

\subsection{Localisations}

Les nodules sont retrouvés principalement dans la peau des membres et de la moitié inférieure du tronc.

Un tableau permet de détailler les localisations relevées dans un cas d'infestation massive :

\begin{tabular}{|c|c|c|}
\hline Tête & & ++ \\
\hline Encolure & & + \\
\hline \multicolumn{3}{|l|}{ Tronc : } \\
\hline & - moitié supérieure & $\mathrm{O}$ \\
\hline & — moitié inférieure & +++ \\
\hline & — ombilic & ++ \\
\hline & - mamelle & + \\
\hline & — périnée & $\mathrm{O}$ \\
\hline \multicolumn{3}{|c|}{ Membre thoracique: } \\
\hline & — épaule & ++ \\
\hline & - bras & ++ \\
\hline \multicolumn{3}{|c|}{ Membre pelvien: } \\
\hline & - fesse & ++ \\
\hline & - cuisse & ++ \\
\hline & - jambe & ++ \\
\hline
\end{tabular}

\subsection{Etude microscopique}

Les nodules contiennent une ou rarement deux femelles, accompagnées de un à sept mâles. Dans un cas, ont même été trouvés dans un seul nodule deux femelles et treize mâles.

La paroi fibreuse des nodules est analogue à celle du type précédent, bien que dans certains cas elle soit beaucoup moins épaisse. 


\section{Discussion}

\subsection{Relations avec $O$, dukei}

Il peut paraître surprenant de trouver simultanément, dans les mêmes localités, deux onchocercoses bovines différentes, toutes deux localisées aux parties inférieures du corps.

Cependant ces deux types se distinguent très bien :

- par les caractères morphologiques des vers adultes et des microfilaires (2);

- par la localisation intradermique des nodules dus à $O$. dermata, alors qu'ils semble que $O$. dukei ne se retrouve jamais à l'intérieur du cuir.

\subsection{Relations avec $O$. ochengi}

En 1969, a été décrite une première onchocercose dermique des bovins, due à $O$. ochengi Bwangamoi, 1969, dans divers pays d'Afrique orientale (Ouganda, Kenya, Ethiopie).

Mais elle aussi se distingue nettement à l'infestation à $O$. dermata :

- par les caractères morphologiques des microfilaires;

- par la localisation prédominante des nodules à $O$. ochengi dans la peau du scrotum et des mamelles.

\subsection{Confusions avec la démodécie bovine}

L'ouverture de certains nodules dermiques a permis de découvrir, au lieu des nématodes attendus, d'innombrables Demodex bovis.
Il serait intéressant d'étudier le rôle respectif de ces deux types de parasites dans les dégâts causés aux cuirs à usage industriel.

\section{CONCLUSIONS}

Au Togo, les bovins (taurins autochtones et zébus provenant de Haute-Volta), outre les infestations de la paroi aortique par $O$. armillata et du ligament cervical par $O$. gutturosa, présentent très fréquemment aussi :

- une onchocercose musculaire, due à $O$. $d u$ $k e i$, et caractérisée par la présence de nodules blanchâtres, d'environ $10 \mathrm{~mm}$ de long, qu'il convient de distinguer à l'abattoir des lésions de cysticercose;

- une onchocercose dermique, due à $O$. dermata, également à caractère nodulaire, mais souvent beaucoup plus difficile à déceler.

\section{Remerciements}

Nous tenons à remercier bien sincèrement tous ceux qui, au Togo, nous ont permis et facilité la réalisation de ce travail, et plus particulièrement :

- Monsieur le Recteur JOHNSON, de l'Université du Bénin;

- Monsieur le Docteur-Vétérinaire SALAMI, Directeur du Service de l'Elevage du Togo;

- Monsieur le Professeur BOURGAT, Directeur de l'Ecole Supérieure d'Agronomie de Lomé.

\section{SUMMARY}

\section{Onchocercosis of Togoland cattle}

The Togoland cattle very frequently catch two nodular onchocercosis : a muscular and subcutaneous one caused by $O$. dukei, Bain, Bussieras and Amégée, 1974; and a dermic one, caused by $O$. dermata Bain, Bussieras and Amégée, 1974.

\section{RESUMEN}

Las oncocercosis de los bovinos causadas por $O$, dukei y $O$. dermata en Togo

Dos oncocercosis con carácter nodular atacan muy frecuentemente los bovinos de Togo: una oncocercosis muscular y subcutánea causada por $O$. dukei Bain, Bussieras y Amégée, 1974; y una oncocercosis dérmica causada por $O$. dermata Bain, Bussieras et Amégée, 1974. 


\section{BIBLIOGRAPHIE}

1. AMEGEE (E.). Les onchocercoses bovines en Afrique. Etude de deux formes nodulaires à $O$. dukei et $O$. dermata au Togo. Thèse DoctoratVétérinaire, Université de Dakar, 1974.

2. BAIN (O.). BUSSIERAS (J.) et AMEGEE (E.). Dualité d'Onchocerca volvulus de l'homme et d'O. sp. Cameron, 1928, du bétail. Nouvelles espèces d'onchocerques bovines au Togo. C.R. Acad. Sci. Paris, série D, 1974, 278 (3) : 369-372.

3. BEAL (W. P. B.). Gold Coast Colony. Report on the Veterinary Department for the years 1927-28 and 1928-29. Accra, 1929.

4. BWANGAMOI (O.). Onchocerca ochengi new species, an intradermal parasite of cattle in East africa. Bull. epizoot. Dis, Afr., 1969, 17 (3) : 321335.

5. BWANGAMOI (O). Dermatitis in cattle caused by Onchocerca ochengi Bwangamoi, 1969, and the effect of the adult filaria on the finished leather. Bull. epizoot. Dis. Afr., 1969, 17 (4) : 435-445.

6. CAMERON (T. W. M.). On a species of Onchocerca from the ox in West Africa. J. Helm., 1928, 6 (3) : 161-164.

7. CAMERON (T. W. M.). The parasites of domestic animals. Londres, Black éd., 1951.

8. HEEVER (L. W. VAN DEN). Deep muscular onchocerciasis. J.S. Afr. vet. Med. Ass., 1971, $42(2): 202$.

9. LE ROUX (P. L.). Report to the Government of the Federation of Rhodesia and Nyasaland on the control of parasitic diseases in livestock. Rome, F.A.O., 1957 (Report no 696).

10. STEWART (J.L.). Gold Coast Colony. Report on the Veterinary Department for the year 1929-30. Accra, 1930. 